\title{
AiMT
}

Advances in Military Technology

Vol. 13, No. 2 (2018), pp. 277-290

ISSN 1802-2308, eISSN 2533-4123

DOI 10.3849/aimt.01236

\section{Modelling and Simulation of Surface-to-Surface Missile General Platform}

\author{
M. K. Elbaioumy ${ }^{1}$, M. M. Elkhatib ${ }^{2 *}$ and A. E. Khalifa ${ }^{1}$ \\ ${ }^{1}$ Alexandria University, Alexandria, Egypt \\ ${ }^{2}$ Military Technical College, Cairo, Egypt
}

The manuscript was received on 26 February 2018 and was accepted after revision for publication on 3 December 2018.

\begin{abstract}
:
In this paper, a general platform of a surface-to-surface missile model is presented. An accurate missile system model was made with consideration of the rigid body dynamics in space with six degrees of freedom and equation of motion starting from Newton second law to the non-linear state space model of the missile. Computer simulations have been executed using the MATLAB/Simulink software. Verification of the system model has been made.
\end{abstract}

\section{Keywords:}

missile modelling, surface-to-surface missile, 6 degrees of freedom, equation of motion, non-linear state space model.

\section{Introduction}

Modelling of flight dynamics is limited to some constraints, such as picking a frame reference coordinate, and derivation of dominant equations of motion. In this derivation, the vehicle is treated as a rigid body when considering its rotational motion, which reduces the degrees of freedom from infinite to only six. The equations of motion can be described in two groups; kinematic equations and dynamic/kinetic equations [1].

The aerodynamic and propulsion force and moment vectors are obtained from Newton's laws of motion and gravity, as well as the aerothermodynamics principles [2]. A MathWork's Simulink software toolbox; Aerospace Block-Set [1] has been used to construct the flight model including: 6 degrees-of-freedom equations of motion, linearization of aerodynamics, also a turbofan engine block, $2^{\text {nd }}$ and $3^{\text {rd }}$ order actuators, several standard atmosphere and earth gravity models, and finally the wind disturbance models [1].

\footnotetext{
* Corresponding author: Department of Electronic Engineering, Military Technical College, Cairo,Egypt,melkhatib@mtc.edu.eg.
} 


\begin{tabular}{|c|c|c|c|}
\hline \multicolumn{4}{|c|}{ Nomenclature } \\
\hline$A, B, C$ & State-space matrices & $N_{\mathrm{v}}$ & Yawing moment due to side velocity \\
\hline$a_{\mathrm{n}}$ & Normal acceleration & $N_{\zeta}$ & Yawing moment due to rudder angle \\
\hline $\mathrm{a}_{\mathrm{nc}}$ & Commanded normal acceleration & $P$ & Position vector \\
\hline B & $\begin{array}{l}\text { Transformation matrix from Earth axes } \\
\text { to body axes }\end{array}$ & $p, q, r$ & $\begin{array}{l}\text { Roll, pitch and yaw rates } \\
\text { Dynamic pressure }\end{array}$ \\
\hline $\mathrm{C}_{\mathrm{D}}$ & Coefficient of drag & $\mathrm{q}_{\mathrm{c}}$ & Commanded pitch rate \\
\hline $\mathrm{C}_{\mathrm{L}}$ & Coefficient of lift & $S$ & Reference area \\
\hline $\mathrm{C}_{\mathrm{l}}$ & Coefficient of rolling moment & $\mathrm{s}$ & Laplace operator \\
\hline $\mathrm{C}_{\mathrm{m}}$ & Coefficient of pitching moment & $\mathrm{T}_{\mathrm{s}}$ & Sampling time \\
\hline $\mathrm{C}_{\mathrm{n}}$ & Coefficient of yawing moment & $\mathrm{t}$ & Time \\
\hline Схвувив & Body axes & $t_{\mathrm{r}}$ & Rise time \\
\hline $\mathrm{C}_{Y}$ & Coefficient of side force & $t_{\mathrm{s}}$ & Settling time \\
\hline$D$ & Drag force, Missile diameter & $u, v, w$ & Velocity component in body axes \\
\hline $\mathrm{e}_{\mathrm{q}}$ & Error in pitch rate & $u$ & Input signal \\
\hline$F_{\mathrm{B}}$ & $\begin{array}{l}\text { Resultant of external forces w.r.t. }{ }^{1} \\
\text { body axes }\end{array}$ & $u_{0}$ & Undisturbed longitudinal velocity \\
\hline$g$ & Gravitational acceleration & $u_{\eta}$ & Control signal \\
\hline$H$ & Altitude & $V$ & Total velocity \\
\hline & Angular momentum w.r.t. body axes & $v_{\mathrm{B}}$ & Velocity w.r.t. body frame \\
\hline $\boldsymbol{I}_{\mathrm{xx}}, \boldsymbol{I}_{\mathrm{yy}}, \boldsymbol{I}_{\mathrm{zz}}$ & Moments of inertia about body axes & $\mathrm{X}_{\mathrm{u}}$ & Axial force due to longitudinal velocity \\
\hline $\boldsymbol{I}_{\mathrm{xy}}, \boldsymbol{I}_{\mathrm{zy}}, \boldsymbol{I}_{\mathrm{xz}}$ & $\begin{array}{l}\text { Product of moments of inertia about } \\
\text { body axes }\end{array}$ & $\mathrm{x}, \mathrm{y}, \mathrm{z}$ & Position of body c.g. w.r.t. Earth axes \\
\hline $\mathrm{i}_{\mathrm{B}}, \mathrm{j}_{\mathrm{B}}, \mathrm{k}_{\mathrm{B}}$ & Body axes unit vectors & $Y$ & Side force \\
\hline & Inertia tensor & $\mathrm{Y}_{\mathrm{r}}$ & Side force due to yaw rate \\
\hline $\mathrm{K}_{\mathrm{a}}$ & Normal acceleration gain & $\mathrm{Y}_{\mathrm{v}}$ & Side force due to side velocity \\
\hline $\mathrm{K}_{\mathrm{q}}$ & Pitch rate gain & $\mathrm{Y}_{\zeta}$ & Side force due to rudder angle \\
\hline $\mathrm{k}$ & Sample number & $\mathrm{y}$ & Output vector \\
\hline$L$ & Lift force, Rolling moment & $\mathrm{Z}_{\mathrm{q}}$ & Normal force due to pitch rate \\
\hline$L_{\mathrm{p}}$ & Rolling moment due to roll rate & $\mathrm{Z}_{\mathrm{w}}$ & Normal force due to vertical velocity \\
\hline$L \xi$ & Rolling moment due to aileron angle & $Z_{\alpha}$ & Normal force due to angle of attack \\
\hline $1_{\mathrm{p}}$ & $\begin{array}{l}\text { Position of accelerometer in front of } \\
\text { c.g. }{ }^{2}\end{array}$ & $Z_{\eta}$ & Normal force due to elevator angle \\
\hline M & $\begin{array}{l}\text { Pitching moment, Resultant of external } \\
\text { moments w.r.t. body axes }\end{array}$ & $\alpha$ & Angle of attack \\
\hline M. O. & Maximum percentage overshoot & $\beta$ & Side slip angle \\
\hline Ма & Mach number & $\zeta$ & Rudder angle \\
\hline $\boldsymbol{M}_{\mathrm{q}}$ & Pitching moment due to pitch rate & $\zeta_{\mathrm{sp}}$ & Short period mode damping ratio \\
\hline $\boldsymbol{M}_{\mathrm{w}}$ & Pitching moment due to vertical velocity & $\eta$ & Elevator angle \\
\hline$M_{\alpha}$ & Pitching moment due to angle of attack & $\theta$ & Pitch angle \\
\hline$M_{\eta}$ & Pitching moment due to elevator angle & $\xi$ & Aileron angle \\
\hline$m$ & Missile mass & $\rho$ & Air density \\
\hline$N$ & Yawing moment & $\varphi$ & Roll angle \\
\hline $\mathrm{N}_{2}$ & Prediction horizon & $\psi$ & Yaw angle \\
\hline$N_{\mathrm{r}}$ & Yawing moment due to yaw rate & $\omega_{\mathrm{B}}$ & Angular velocity w.r.t. body frame \\
\hline $\mathrm{Nu}_{\mathrm{u}}$ & Control horizon & $\omega_{\mathrm{n}}$ & Short period mode natural frequency \\
\hline
\end{tabular}

\footnotetext{
${ }^{1}$ w.r.t. - with respect to

2 c.g. - centre of gravity
} 


\section{Reference Frames}

\subsection{Earth-Centred Inertial (ECI) Reference Frame $\left\langle\mathrm{OX}_{\mathrm{c}} \mathrm{Y}_{\mathrm{c}} \mathrm{Z}_{\mathrm{c}}\right\rangle$}

This is a coordinate frame with its origin at the Earth's centre, which moves with the Earth, but with a fixed orientation relative to the stars [3].

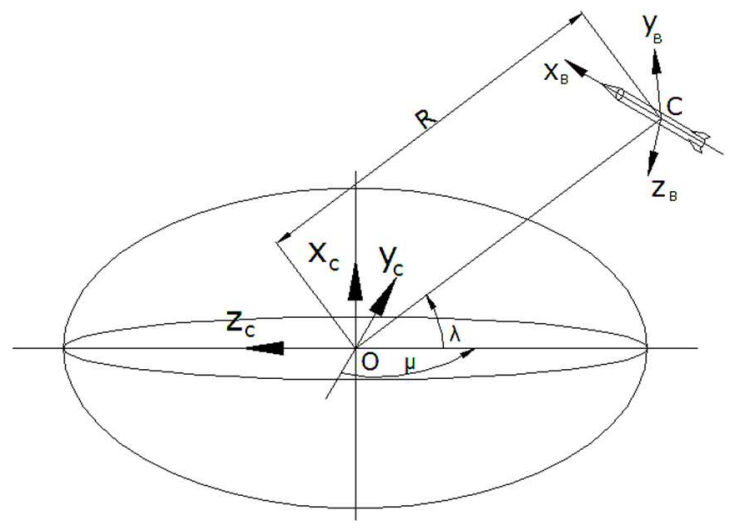

Fig. 1 Coordinate frames

\subsection{Body-fixed Reference Frame $\left\langle\mathrm{CX}_{\mathrm{B}} \mathrm{Y}_{\mathrm{B}} \mathrm{Z}_{\mathrm{B}}\right\rangle$}

This is a coordinate frame which is located at the vehicle centre of gravity with origin $\mathrm{C}$ as shown in Fig. 1. The frame moves with the flying vehicle and the $\mathrm{X}$-axis is directed towards the longitudinal axis of the vehicle, the Y-axis is directed towards the lateral axis rightward from the rear and the $\mathrm{Z}$-axis is directed downwards to complete the orthogonal tetrahedral frame Fig. 2, [3].

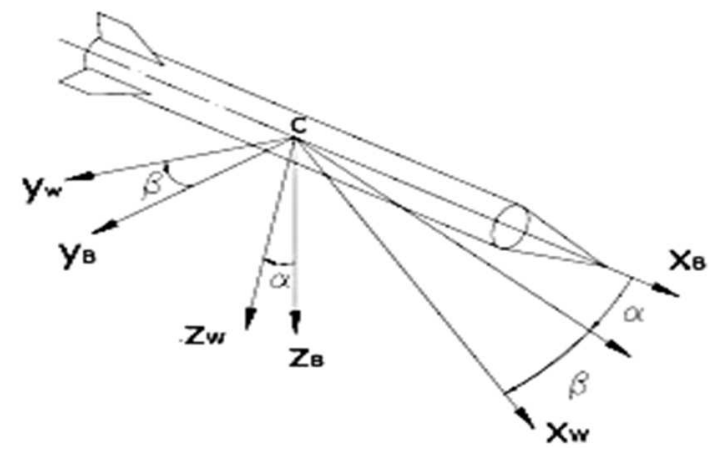

Fig. 2 Missile reference frames: body-fixed reference frame and wind-axes reference frame Heading

\subsection{Wind-axes Reference Frame $\left\langle\mathrm{CX}_{\mathrm{w}} \mathrm{Y}_{\mathrm{w}} \mathrm{Z}_{\mathrm{w}}\right\rangle$}

This is a coordinate frame that is located at the vehicle centre of gravity. The frame moves with the flying vehicle and the $\mathrm{X}_{\mathrm{w}}$-axis is directed against the free stream velocity vector. This frame results from rotating the body-fixed reference frame with an angle of attack $\alpha$ around the negative $\mathrm{Y}_{\mathrm{B}}$-axis and then from the rotation around the resulted $\mathrm{Z}$ axis with side slip angle $\beta$, as shown in Fig. 2. 


\subsection{Firing point Reference Frame $\left\langle\mathrm{OX}_{\mathrm{f}} \mathrm{Y}_{\mathrm{f}} \mathrm{Z}_{\mathrm{f}}\right\rangle$}

The axes are fixed on the Earth and rotate with it while the origin is placed on the firing point. The $\mathrm{OX}_{\mathrm{f}}$ axis is taken in the direction of firing direction and the axis $\mathrm{OZ}_{\mathrm{f}}$ tends to be directed downward vertically. Namely, it takes the direction of the gravity, and $\mathrm{OY}_{\mathrm{f}}$ is taken so that the coordinates establish the right-handed system [3], as shown in Fig. 3.

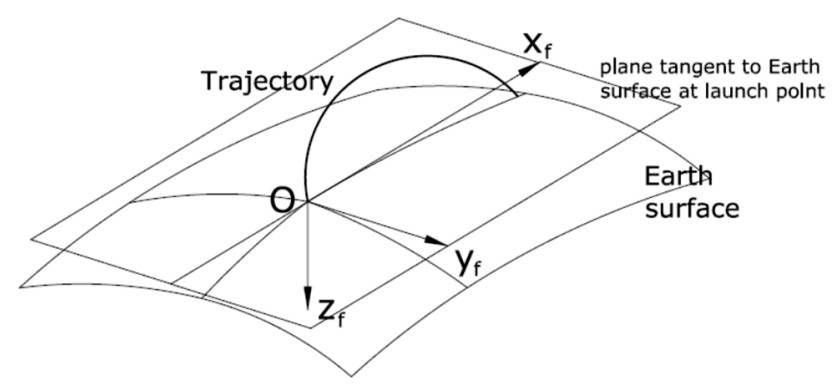

Fig. 3 Firing-point reference frame

It is necessary to choose an inertial reference frame (which is considered fixed) in the development of the vehicle mathematical model. Inertial frame is considered to be non-rotating and non-accelerating (but it may be moved with constant rectilinear velocity) relative to the average position of the "fixed" distant stars. If the Earth is assumed to be flat and non-rotating, the firing point coordinate system could be considered as the inertial coordinate system [3].

\section{Transformation between Reference Frames}

According to Euler, the orientation of one reference frame with respect to another is completely defined by a set of three successive virtual perpendicular rotations in a specific order. The orientation is then completely defined by the values of the angles of rations called Euler angles and the order of them is widely agreed in aeronautics to be yawing, pitching then rolling [3].

\subsection{Firing point and Body fixed Frames}

This transformation is used to get the components of any vector defined in the firingpoint reference frame into the body-fixed reference frame axes. The transformation matrix results from a sequence of rotations $(\psi, \theta, \phi)$ used with a specific order to describe the instantaneous attitude according to the firing reference frame [3].

$$
\left[\begin{array}{l}
x \\
y \\
z
\end{array}\right]_{\text {Body }}=\left[\begin{array}{ccc}
\cos \theta \cos \psi & \cos \theta \sin \psi & -\sin \theta \\
-\cos \phi \sin \psi+\sin \phi \sin \theta \cos \psi & \cos \phi \cos \psi+\sin \phi \sin \theta \sin \psi & \sin \phi \cos \theta \\
\sin \phi \sin \psi+\cos \phi \sin \theta \cos \psi & -\sin \phi \cos \psi+\cos \phi \sin \theta \sin \psi & \cos \phi \cos \theta
\end{array}\right]\left[\begin{array}{l}
x \\
y \\
z
\end{array}\right]_{\mathrm{f}}=R_{\mathrm{FB}}\left[\begin{array}{l}
x \\
y \\
z
\end{array}\right]_{\mathrm{f}}
$$

\subsection{Wind axes and Body fixed Frames}

This transformation is used to get the components of any vector defined in the windaxes reference frame (e.g. aerodynamic forces) into the body-fixed reference frame axes. The transformation matrix results from a sequence of basis rotations $(\beta, \alpha)$ used to describe the instantaneous orientation of velocity vector starting from the wind frame [3]. 


$$
\left[\begin{array}{l}
x \\
y \\
z
\end{array}\right]_{\mathrm{Body}}=\left[\begin{array}{ccc}
\cos \theta \cos \psi & \cos \theta \sin \psi & -\sin \theta \\
-\cos \phi \sin \psi+\sin \phi \sin \theta \cos \psi & \cos \phi \cos \psi+\sin \phi \sin \theta \sin \psi & \sin \phi \cos \theta \\
\sin \phi \sin \psi+\cos \phi \sin \theta \cos \psi & -\sin \phi \cos \psi+\cos \phi \sin \theta \sin \psi & \cos \phi \cos \theta
\end{array}\right]\left[\begin{array}{l}
x \\
y \\
z
\end{array}\right]_{\mathrm{f}}=R_{\mathrm{FB}}\left[\begin{array}{c}
x \\
y \\
z
\end{array}\right]_{\mathrm{f}}
$$

\section{Equations of Motion}

The vehicle equations of motion can be calculated from Newton's second law of motion. The equations show that the summation of all forces applied externally on a body should be equal to the time rate of change of its momentum. The time rates of change take into consideration the inertial reference frame, under certain hypotheses:

- The intended vehicle is a surface-surface tactical missile utilizing solid propellant engine for propulsion in the form of boost-sustainer motor and the missile is aerodynamically controlled using four all-movable fins in the form of " $X$ "-configuration, as shown in Fig. 2.

- The origin of the body axes system is located at the centre of gravity of the vehicle and fixed to the vehicle and it rotates with it. The axes are taken, as shown in Fig. 2, with $\mathrm{X}_{\mathrm{B}}$ forward from vehicle centre of gravity towards the vehicle nose tip, $\mathrm{Y}_{\mathrm{B}}$ out to the right and $\mathrm{Z}_{\mathrm{B}}$ downward.

- The axes $\mathrm{X}_{\mathrm{B}}$ and $\mathrm{Z}_{\mathrm{B}}$ lie in the plane of symmetry of the vehicle, and axes $\mathrm{X}_{\mathrm{B}}$ and $\mathrm{Y}_{\mathrm{B}}$ lie in the other plane of symmetry. Hence, the cross moments of inertia $I_{\mathrm{xy}}, I_{\mathrm{xz}}$ and $I_{\mathrm{yz}}$ have all zero value. The missile has the same mass distribution in the axes $\mathrm{Y}_{\mathrm{B}}$ and $\mathrm{Z}_{\mathrm{B}}$, so, the second moments of inertia of both axes are equal $\left(I_{\mathrm{yy}}=I_{\mathrm{zz}}\right)$.

- The equations of motion are composed of:

- Dynamic equations: they consist of vector force and moment equations.

- Kinematic equations: they consist of attitude and trajectory equations.

- Although the vehicle mass changes considerably due to the propellant burning in the powered phase and it remains constant after engine shut-off, the amount of propellant consumed during certain integration time step in the powered region of the vehicle flight may be safely neglected [1].

\subsection{Force Equations}

The force equation is derived with respect to the body-fixed reference frame to get the acceleration with respect to body frame as shown in the next equations:

$$
\sum \boldsymbol{F}_{\mathrm{B}}=\frac{\mathrm{d}}{\mathrm{d} t}\left(m v_{\mathrm{B}}\right)
$$

From Eq. 3, the force equation can be written in the form:

$$
\dot{\boldsymbol{v}}_{\mathrm{B}}=\frac{1}{m} \boldsymbol{F}_{\mathrm{B}}-\omega_{\mathrm{B}} \times \boldsymbol{v}_{\mathrm{B}}+R \boldsymbol{g},
$$

\subsection{Moment Equations}

To get the equations of angular motion, it is necessary to consider that the moment affecting the vehicle is equal to the changing rate of its angular momentum $\boldsymbol{H}_{\mathrm{B}}$, then:

$$
\boldsymbol{M}_{\mathrm{B}}=\frac{\mathrm{d} \boldsymbol{H}_{\mathrm{B}}}{\mathrm{d} t} .
$$


For fixed controls airframe, the overall moment $\boldsymbol{M}_{\mathrm{B}}$ acting on airframe with respect to body axes resulted from the moment of the resultant aerodynamic force due to the offset of the centre of pressure (the point of action of resultant aerodynamic force) from the centre of mass, in addition to the moment resulting from the thrust force due to the offset between the point of action of the thrust and the centre of mass. The moment equation $[3,4]$ could be written as:

$$
\dot{\omega}_{\mathrm{B}}=I^{-1} \boldsymbol{M}_{\mathrm{B}}+I^{-1}\left(\omega_{\mathrm{B}} \times \boldsymbol{H}_{\mathrm{B}}\right) .
$$

\subsection{Attitude Equations}

The $\boldsymbol{R}$ matrix describes the attitude of the vehicle as it changes with time. Its elements are functions of the three Euler angles and the body-axes rates $(p, q, r)$ produce a corresponding set of attitude angular rates, depending on the particular instantaneous attitude.

The relation between Euler angles derivative and angular velocity of airframe $[3,5]$ could be written as follows:

$$
\left[\begin{array}{c}
\dot{\phi} \\
\dot{\theta} \\
\dot{\psi}
\end{array}\right]=\left[\begin{array}{ccc}
1 & \tan \theta \sin \phi & \tan \theta \cos \phi \\
0 & \cos \phi & -\sin \phi \\
0 & \frac{\sin \phi}{\cos \theta} & \frac{\cos \phi}{\cos \theta}
\end{array}\right]\left[\begin{array}{l}
p \\
q \\
r
\end{array}\right] .
$$

Eq. 7 represents the kinematic relation between changing rate of Euler angles of the vehicle and body angular velocity. Eq. 7 has been commonly used for aircraft simulation, but it is not suitable for around-the-Earth flight, all-attitude flight, and simulation of spinning bodies.

\subsection{Navigation Equations}

The location of the vehicle mass centre relative to the Earth is given by the spherical polar coordinates $\boldsymbol{R}$ (geocentric radius), $\mu$ (longitude) and $\lambda$ (latitude), as shown in Eq. 2. But, in case of calculation of position of vehicle mass centre relative to an inertial frame (firing-plane frame), the position with respect to the inertial frame is the integration of the velocity in that frame, i.e.:

$$
\left[\begin{array}{c}
\dot{x}_{e} \\
\dot{y}_{e} \\
\dot{z}_{e}
\end{array}\right]=\boldsymbol{R}\left[\begin{array}{c}
u \\
v \\
w
\end{array}\right] .
$$

\subsection{Wind Equations}

The aerodynamic forces on vehicle are created by its motion relative to the surrounding air. In case of existence of wind (atmosphere is not at rest), the air itself is considered in motion relative to the inertial reference frame. It is assumed that the local wind has north, east, and down velocity components $V w_{x}, V w_{y}$ and $V w_{z}$, respectively, in the firing plane. Then the absolute velocity of the missile with respect to the firing-plane is the vector sum of the velocity of missile with respect to the air $V_{\mathrm{R}}$ and the velocity of wind with respect to the firing-plane $w_{\mathrm{f}}$ transformed to body-frame [2]. The velocity of the vehicle centre-of-mass with respect to the air is then given by: 


$$
\boldsymbol{v}_{\mathrm{R}}=\boldsymbol{v}_{\mathrm{B}}-\boldsymbol{R}_{\mathrm{FB}} \boldsymbol{w}_{\mathrm{f}}=\left[\begin{array}{c}
u \\
v \\
w
\end{array}\right]-\boldsymbol{R}_{\mathrm{FB}}\left[\begin{array}{c}
V w_{x} \\
V w_{y} \\
V w_{z}
\end{array}\right]_{\mathrm{f}}=\left[\begin{array}{c}
u \\
v \\
w
\end{array}\right]-\boldsymbol{R}_{\mathrm{FB}} \boldsymbol{w}_{\mathrm{f}} \text {. }
$$

\section{Missile Simulation}

The core of the simulation is a non-linear model of the vehicle dynamics which includes twelve Ordinary non-linear Differential Equations (ODE's or state equations) which are Eqs 4, 6, 7, 8, and a large number of equations of output. This model can be broken down into a number of different modules which are almost independent from the kind of moving vehicle under consideration.

\subsection{Vehicle characteristics and flight conditions}

For calculating the dynamic trajectory [6], the airframe of the vehicle is specified with a hypothetical frame of cruciform shape, conical head and four all-movable tail fins of X-configuration, as shown in Fig. 4.

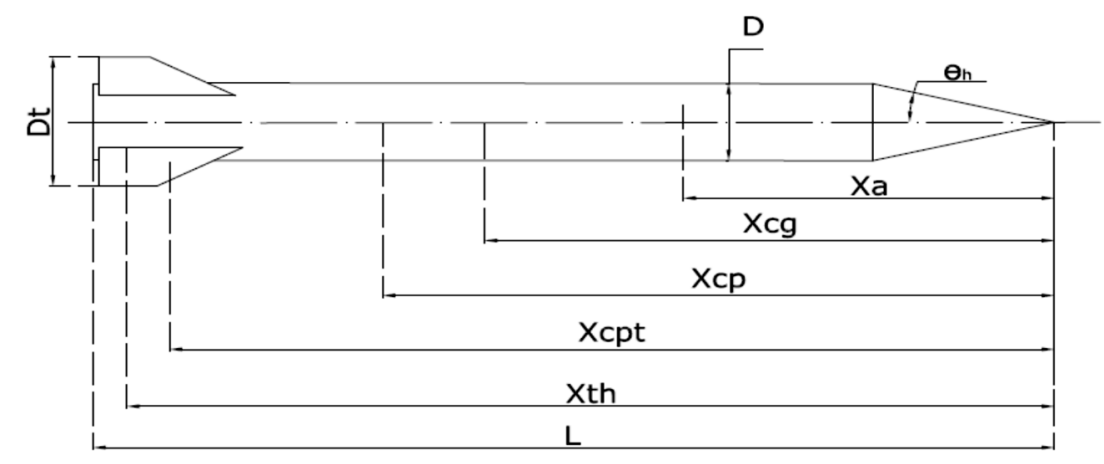

Fig. 4 Model configuration and main dimensions

The indicated dimensions are defined as follows:

$D$...... the missile diameter

L........ the missile total length

$X_{\mathrm{cg}} \ldots .$. the centre of gravity position

$X_{\mathrm{cp}} \ldots . .$. the centre of pressure of the missile

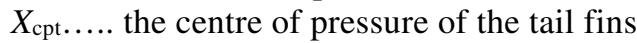

$D_{\mathrm{t}} \ldots \ldots$ the span of the tail fins

$\Theta_{\mathrm{h}} \ldots . .$. the angle of inclination of the warhead

$X_{\mathrm{a}} \ldots \ldots$ the position of the accelerometer measured from nose tip

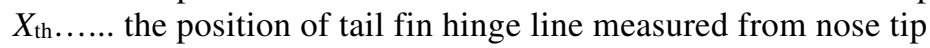

\subsection{Thrust}

A general form of the thrust equation for the rocket motor of solid propellant $[7,8]$ is given by:

$$
T=\dot{m} V_{\text {exit }}+\left(P_{e}-P_{a}\right) A_{e}
$$


From Eq. 10, the thrust $T_{0}$ at sea level can be written as:

$$
T_{0}=\dot{m} V_{\text {exit }}+\left(P_{e}-P_{0}\right) A_{e} .
$$

where $P_{0}$ is the atmospheric pressure at sea level.

The thrust $T_{H}$ at certain altitude $H$ is given by:

$$
T_{H}=\dot{m} V_{\text {exit }}+\left(P_{e}-P_{H}\right) A_{e} .
$$

This equation yields:

$$
T_{H}=\dot{m} V_{\text {exit }}+\left(P_{e}-P_{0}\right) A_{e}+\left(P_{0}-P_{H}\right) A_{e} .
$$

Finally:

$$
T_{H}=T_{0}+\left(P_{0}-P_{H}\right) A_{e} .
$$

Then, from the resulting data of rocket motor static test at sea level $T_{0}$ and the pressure at certain altitude $H$, the thrust at that altitude $T_{H}$ can be calculated.

\subsection{Atmosphere}

According to ISA model, the air temperature $T$ changes with altitude [1,9] as follows:

$$
T= \begin{cases}288.15-0.0065 H & \text { for altitudes from sea level to } 11000 \mathrm{~m} \\ 216.65[\mathrm{~K}] & \text { for altitudes from } 11000 \mathrm{~m} \text { to } 20000 \mathrm{~m} \\ 216.65+0.001(H-20000) & \text { for altitudes from } 20000 \mathrm{~m} \text { to } 32000 \mathrm{~m} \\ 228.65+0.0028(H-32000) & \text { for altitudes from } 32000 \mathrm{~m} \text { to } 47000 \mathrm{~m}\end{cases}
$$

Where $T[\mathrm{~K}]$ is the temperature at altitude $H[\mathrm{~m}]$ and $T_{0}$ is the temperature at sea level and it equals $288.15 \mathrm{~K}$.

Then the atmosphere layers will be divided into two types:

a) Gradient layer in which the temperature changes with factor $\tau_{d}$

b) Isothermal layer in which the temperature remains constant $\left(\tau_{d}=0\right)$

$$
\tau_{d}= \begin{cases}-0.0065[\% \mathrm{~m}] & \text { for } H \text { from } 0 \text { to } 11000 \mathrm{~m} \\ 0 & \text { for } H \text { from } 11000 \mathrm{~m} \text { to } 20000 \mathrm{~m} \\ 0.001[\% \mathrm{~m}] & \text { for } H \text { from } 20000 \mathrm{~m} \text { to } 32000 \mathrm{~m} \\ 0.0028[\% \mathrm{~m}] & \text { for } H \text { from } 32000 \mathrm{~m} \text { to } 47000 \mathrm{~m}\end{cases}
$$

The equation of atmospheric pressure can be derived to be $[1,9]$ :

- For gradient layer

$$
P=P_{d}\left(\frac{T}{T_{d}}\right)^{-\frac{g_{0}}{R \tau_{d}}} .
$$

- For isothermal layer

$$
P=P_{d} \mathrm{e}^{-\frac{g_{0}}{R T_{d}}\left(H-H_{d}\right)} .
$$

The density is given by:

- Gradient layer 


$$
\rho=\rho_{d}\left(\frac{T}{T_{d}}\right)^{-\left(\frac{g_{0}}{R \tau_{d}}+1\right)}
$$

- Isothermal layer

$$
\rho=\rho_{d} \mathrm{e}^{-\frac{g_{0}}{R T_{d}}\left(H-H_{d}\right)} .
$$

The sonic speed at altitude $H$ is given by:

$$
a=\sqrt{\chi R T}=20.05 \sqrt{T},
$$

where $\chi$ is the exponent ratio and it is equal to 1.4 for air [4] and $g_{0}$ is the gravitational acceleration at sea level

\subsection{Aerodynamic Forces and Moments}

The aerodynamic forces are defined in terms of dimensionless coefficients, the flight dynamic pressure and the reference area [5] as follows:

$$
\begin{aligned}
& X=\mathrm{C}_{\mathrm{x}} \frac{1}{2} \rho V^{2} \mathrm{~S}, \\
& Y=\mathrm{C}_{\mathrm{y}} \frac{1}{2} \rho V^{2} \mathrm{~S}, \\
& Z=\mathrm{C}_{\mathrm{z}} \frac{1}{2} \rho V^{2} \mathrm{~S} .
\end{aligned}
$$

The resultant aerodynamic force acts at the point called the centre of pressure and this point is usually not located at the centre of gravity of the vehicle. The aerodynamic moment definition is the same as the aerodynamic forces in terms of dimensionless coefficients, dynamic pressure, reference area and a characteristic length as follows:

$$
\begin{aligned}
& N=\mathrm{C}_{\mathrm{n}} \frac{1}{2} \rho V^{2} S D \Rightarrow \text { Yawing Moment, } \\
& M=\mathrm{C}_{\mathrm{m}} \frac{1}{2} \rho V^{2} S D \Rightarrow \text { Pitching Moment, } \\
& L=\mathrm{C}_{1} \frac{1}{2} \rho V^{2} S D \Rightarrow \text { Rolling Moment. }
\end{aligned}
$$

In case of zero aerodynamic roll, the angle of attack and sideslip angle can be defined in terms of the velocity components [4] as follows:

$$
\begin{aligned}
& \alpha=\arctan \frac{w}{u}, \\
& \beta=\arctan \frac{v}{u}
\end{aligned}
$$

\subsection{Aerodynamic Coefficients}

For simplicity, the aerodynamic forces and moments acting on the aircraft are defined in terms of dimensionless aerodynamic coefficients $C_{x}, C_{y}, C_{z}, C_{1}, C_{m}$, and $C_{n}$ and they are called total coefficients of moments and forces. They depend on the control surfaces deflection, the aerodynamic angles and the angular rates $[3,10]$. 


\subsection{Construction of Simulation}

The open-loop simulation is performed using Matlab / Simulink package. The block diagram is shown in Fig. 5.

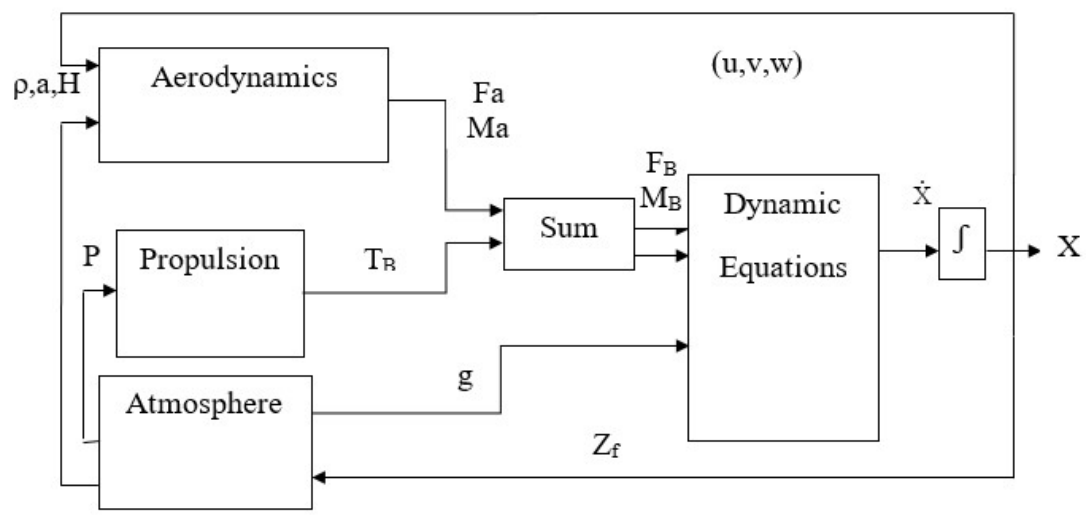

Fig. 5 Block diagram of the vehicle

From Fig. 5, it can be concluded that:

- The control actions are not taken into account.

- Neither the disturbance of the atmosphere is taken into account.

- The vehicle does not contain any sensors and is not guided.

\subsection{OPENLOOP SIMULINK/MATLAB Model}

This part discusses the Simulink / Matlab modelling of Missile system. The model is based on the derivation of the mathematical model that was listed before in the mathematical modelling part. The complete set of equations of motion is coded into Simulink blocks leading to a missile simulator that helps in the control process [11].

\subsubsection{Missile Simulator}

The missile simulator is the main system block and it includes various subsystems. It has 12 output signals representing the position and the orientation of the vehicle and their rates or velocities considering the body reference frame. Each internal subsystem of the missile simulator is illustrated.

\subsubsection{Dynamics Block}

The dynamic block is responsible for producing 12-output control signal that controls the equations of motion. It produces these signals according to the input of the mathematical modelling of the missile and its movements. The input to the block includes the forces on the body, body momentum, mass, mass of inertia, gravity and time respectively.

$$
X_{d}=\operatorname{Dynamics}\left(X, F_{\mathrm{b}}, M_{\mathrm{b}}, m, I_{\mathrm{x}}, I_{\mathrm{y}}, m_{\mathrm{dot}}, g, t\right) .
$$

\subsubsection{Aerodynamics Block}

Aerodynamics block is responsible for calculating the body forces and momentums for the dynamics block as an output to control the missile body according to the input signals; Velocity, Atmosphere density $\rho$, Acceleration, Height and Centre of gravity respectively. 


\subsubsection{Atmosphere Block}

The atmosphere block is responsible for calculating the $\rho$ (atmosphere density), acceleration, gravity, height and the pressure at certain level as an output signal according to the position of the missile as an input.

$$
[\rho, a, g, H, P]=\text { Atmosphere }[\mathrm{Pos}] .
$$

\subsubsection{Propulsion Block}

The propulsion block is responsible for calculating the thrust force needed for the dynamic block according to the calculated pressure. The missile parameters like the centre of gravity and mass of inertia are fed as inputs to the block.

\subsection{Results of Simulation}

Fig. 6 shows the results of simulation performed on Matlab/Simulink package.
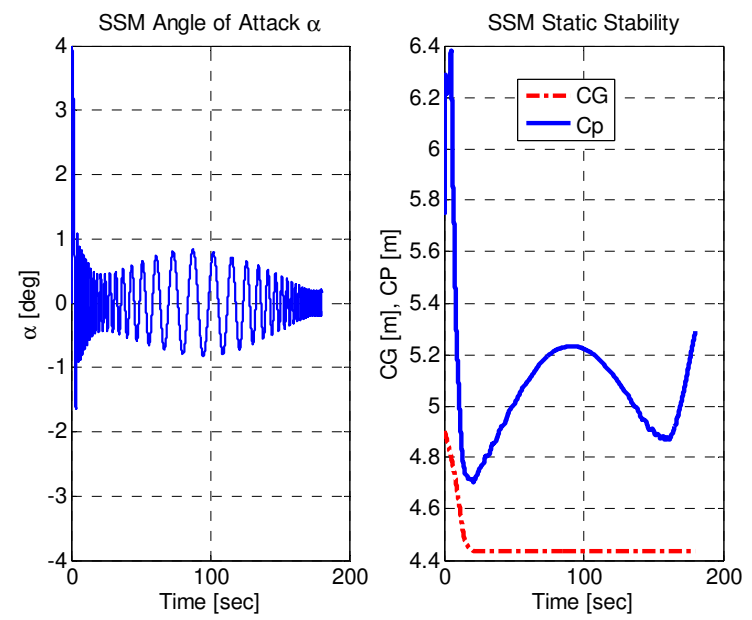

Fig. 6a Results of open-loop simulation - Angle of attack response and static stability

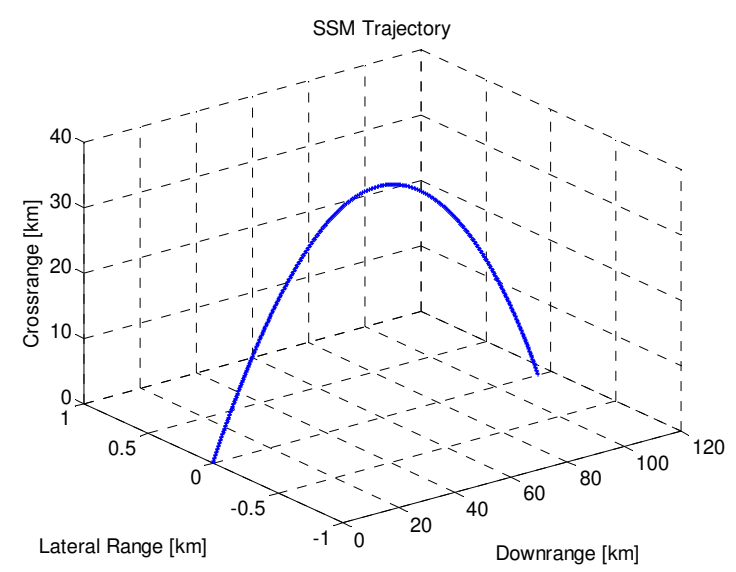

Fig.6b Results of open-loop simulation - The trajectory of uncontrolled missile in inertial frame 

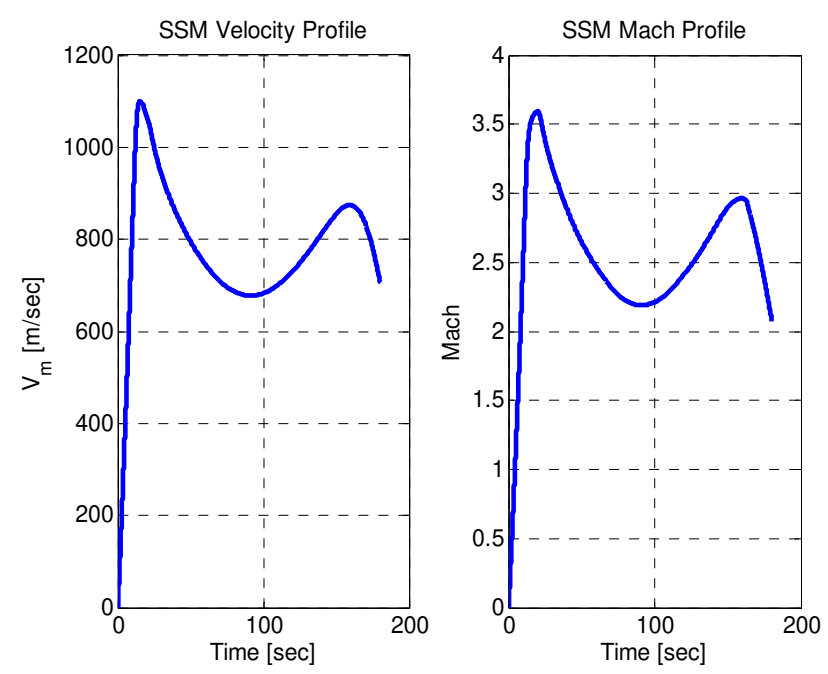

Fig. 6c Results of open-loop simulation - Change of value of velocity and Mach number

\subsection{Flight Path with Different Launching Angles}

Changing of the launching angle will directly affect the range and the summit of the missile trajectory due to the change of the initial pitch angle $\theta_{0}$ which is one of the initial conditions [12]. The previous results of simulation are calculated at launching angle $60^{\circ}$. In order to study the launching angle effect on various parameters, simulation is performed at different launching angles and then the trajectories of uncontrolled missile are drawn at the angles $40^{\circ}, 45^{\circ}, 50^{\circ}, 55^{\circ}, 60^{\circ}, 65^{\circ}, 70^{\circ}, 80^{\circ}$, as shown in Fig. 7. The results clarify that the range increases with the launching angle till $65^{\circ}$ and then decreases with angle increasing. It is concluded that the maximum range is obtained at launching angle $\theta_{0}=60^{\circ}-65^{\circ}$, but increasing of launching angle increases the summit and it is preferred to keep the missile in the dense atmosphere in order to attain a quiet aerodynamic control force created by fins. Thus, it was suitable to choose the launching angle to be $60^{\circ}$.

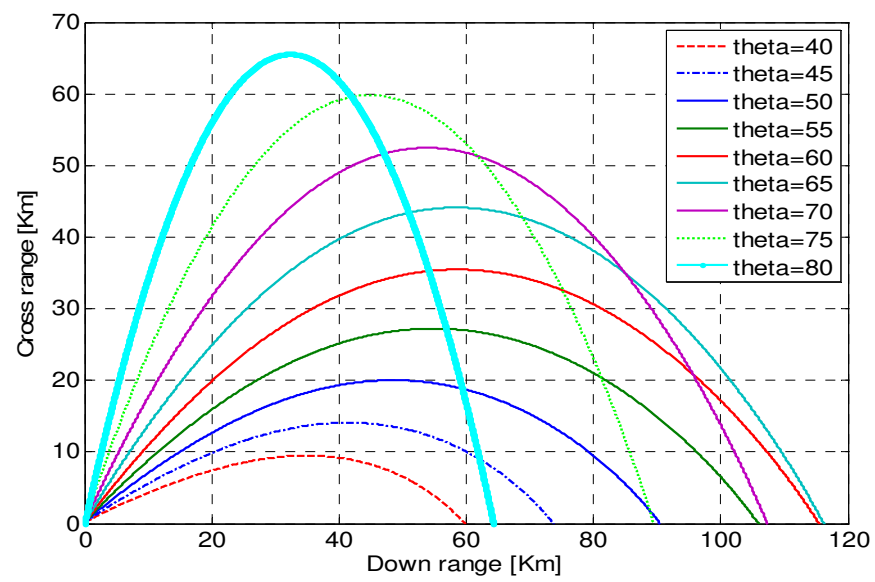

Fig. 7. Trajectory with different launching angles 
Based on the results, it can be concluded that:

- The total flight time of missile is $180.2 \mathrm{~s}$.

- The max. altitude reached by the missile is $35.5 \mathrm{~km}$.

- The total distance travelled by the missile on a flat Earth along inertial frame $X$ axis is $115.5 \mathrm{~km}$.

- The powered phase is considered to be terminated at the beginning of a velocity decrease due to motor burnout. The beginning of the decrease occurs at the time $(t=13.05 \mathrm{~s})$. So, the powered phase will be from the time ( $t=0$ to $13.05 \mathrm{~s})$ and the unpowered phase will be from the time ( $t=13.05 \mathrm{~s}$ till the end of flight).

- From Fig. 6a, which describes centre of gravity and centre of pressure change with time, the missile will remain statically stable during the whole flight time since the centre pressure remains behind centre of gravity.

- In Fig. 6, the response of the angle of attack with respect to the time mainly depends on the undamped natural frequency, damping ratio of short period mode $\left(\omega_{s p}, \zeta_{s p}\right)$, and slightly on undamped natural frequency and damping ratio of phugoid mode $\left(\omega_{p h}, \zeta_{p h}\right)[13]$.

- The undamped natural frequency of short period mode $\omega_{s p}$ depends mainly on pitching moment because of the angle of attack derivative $M_{\alpha}$. The damping ratio $\zeta_{s p}$ depends on pitching moment due to the pitch rate derivative $M_{q}$ and normal force due to the angle of attack derivative $Z_{\alpha}$ [3].

- The frequency of the angle of attack response increases rapidly in the powered phase due to the rapid increase of pitching moment because of angle of attack derivative $M_{\alpha}$ in the same phase. Then the frequency decreases at the beginning of the unpowered phase due to the decrease of the same derivative. Then it returns to the increase as the dynamic pressure increases and then the derivative increases as well.

- The damping ratio of the angle of attack response increases rapidly in the powered phase due to the rapid increase of both pitching moments because of the pitch rate derivative $M_{q}$ and normal force because of angle of attack derivative $Z_{\alpha}$ in the same phase. Then, the damping ratio decreases at the beginning of the unpowered phase due to decrease of the both same derivative. Then, it returns to the increase as the dynamic pressure increases and then the derivatives increase, too.

\section{Model Verification}

The model has been verified with real recorded telemetry data with the Russian missile type " $9 k 52$ Luna". Additionally, it has been verified with trajectory/projectiles equations, to hit a target at altitude $y$ and range $x$ when fired from point $(0,0)$ considering the initial speed $v$ so the required angle(s) of launch are:

$$
\theta=\arctan \left(\frac{v^{2} \pm \sqrt{v^{4}-g\left(\mathrm{~g} x^{2}+2 y v^{2}\right)}}{g x}\right) .
$$

The square roots of Eq. 24 refer to the two potential launching angles, provided that they are not imaginary. This formula is used to find the desired launching angle for model verification such that the desired altitude reached by the missile is $35.5 \mathrm{~km}$ and 
the distance travelled by the missile along the flat earth is $115.5 \mathrm{~km}$. By substitution we will find out that the launching angle is $60^{\circ}$.

\section{Conclusion}

In this paper, a general platform of a surface-to-surface missile model is presented, focusing on discussing the system dynamics and Simulink model. An accurate missile system model was made with consideration of the rigid body dynamics in space with six degrees of freedom and equation of motion starting from Newton second law to the non-linear state space model of the missile. Computer simulations have been executed using the MATLAB / Simulink software. Verification of the system model has been made. The system response shows a good result after testing under various input signals.

\section{References}

[1] OGATA, K. Modern Control Engineering. Pearson Education, $4^{\text {th }}$ ed. 2002.

[2] LIN, C.M. and PENG, Y.F. Missile Guidance Law Design Using Adaptive Cerebellar Model Articulation Controller. IEEE Transactions on Neural Networks, 2005 vol. 16, no. 3. ISSN 1045-9227. DOI 10.1109/TNN.2004.839358.

[3] STEVENS, B. and LEWIS, F. Aircraft Control and Simulation. John Wiley, 1992.

[4] BLAKELOCK, J.H., Automatic Control of Aircraft and Missiles. John Wiley, $2^{\text {nd }}$ ed. 1991.

[5] NELSON, R. Flight Stability and Automatic Control. McGraw-Hill, 1989.

[6] SIOURIS, G.M. Missile Guidance and Control Systems. Springer, 2004.

[7] SANAGAVARAPU, S.R. et al. Mathematical Modeling and Simulation of Missile Target Engagement - 6 DOF Simulation using PN Guidance. Journal of Basic and Applied Engineering Research, 2015, vol. 2, no. 7. ISSN 2350-0077.

[8] BARRERE, M. et al. Rocket Propulsion. Elsevier, 1960.

[9] GOLNARAGHI, F. and KUO, B. Automatic Control Systems. Complex Variables, 2010, $9^{\text {th }}$ ed. vol. 2.

[10] TEWARI, A. Atmospheric and Space Flight Dynamics. Springer, 2007.

[11] RAUW, M. FDC 1.2 - A Simulink Toolbox for Flight Dynamics and Control Analysis. 2001.

[12] WANG, Q. and WANG, L.M. Research on Simulation System of Missile and Arrows Based on Unity3D. In $2^{\text {nd }}$ International Conference on Computational Modeling, Simulation and Applied Mathematics (CMSAM 2017). Shenzhen, 2017.

[13] EL-SHIKH, G.A., An Investigation into the Performance of Strapdown Inertial Navigation. Cairo: Military Technical College, 1990. 